\title{
Optical properties of X-ray selected stars in NGC 2244 in the Rosette Nebula
}

\author{
T. W. Berghöfer ${ }^{1}$ and D. J. Christian ${ }^{2}$ \\ 1 Hamburger Sternwarte, Gojenbergsweg 112, 21029 Hamburg, Germany \\ 2 Computer Sciences Corporation, Space Telescope Science Institute, 3700 San Martin Drive, Baltimore, \\ MD 21218, USA
}

Received 29 June 2001 / Accepted 17 January 2002

\begin{abstract}
We present high spatial resolution optical photometry of NGC 2244 in the Rosette Nebula using the NOAO MOSAIC Imager at Kitt Peak. We obtained BVIR and $\mathrm{H} \alpha$ images of the central cluster and surrounding nebulae, and present results for 138 sources selected from X-ray observations with the ROSAT PSPC and HRI. Color-magnitude diagrams of the cluster show a large number of the stars are still undergoing contraction onto the main sequence. The faintest X-ray selected cluster members have the highest X-ray-to-optical luminosities $\left(L_{\mathrm{x}} \approx 7 \times 10^{30} \mathrm{erg} \mathrm{s}^{-1} ; L_{\mathrm{x}} / L_{\mathrm{bol}} \approx 10^{-2}\right.$ to $\left.10^{-3}\right)$ and indicate they are a population of young active late-type stars. $\mathrm{H} \alpha$ emission from the X-ray emitters is also remarkable. We have extended the detections of PMS stars in NGC 2244 well into the range of K spectral types. While most of the cluster stars are located in the colormagnitude diagrams in between the ZAMS and the 3 Myrs isochrone, significantly younger low mass stars exist and confirm earlier reports that star formation is still going on in the Rosette Nebula/NGC 2244 region.
\end{abstract}

Key words. galaxy - open clusters and associations: individual: NGC 2244 - stars: early-type - stars: late-type stars: activity - stars: pre-main sequence - X-ray: stars

\section{Introduction}

Young open clusters provide a laboratory to study a large number of stars with the same origins. Often found embedded in nebula and molecular clouds, young open clusters also provide an excellent place to study star formation for a wide range of initial stellar masses, evolution onto the main sequence, and interactions with the ISM. X-ray observations have shown many young open cluster members to be very active young stars and strong emitters of X-ray radiation. Such X-ray observations have proved an invaluable tool for detecting T Tauri stars (e.g. Wichmann et al. 1996 and references therein).

As far as X-ray observations are concerned nearby young open clusters have all their own difficulties. Large reddening by the interstellar medium or internal cluster gas makes it rather difficult to detect X-ray emission of stars in these clusters. Another problem of nearby clusters is the separation between older foreground stars and cluster members, which is often difficult to achieve.

Send offprint requests to: T. Berghöfer, e-mail: thomas.berghoefer@desy.de

* Table 3 is only available in electronic form at the CDS via anonymous ftp to cdsarc.u-strasbg.fr (130.79.128.5) or via

http://cdsweb.u-strasbg.fr/cgi-bin/qcat?J/A+A/384/890
For instance, the 2-3Myrs old star cluster associated with the Orion Nebula has been extensively studied with ROSAT (Gagné et al. 1995). This region is located at a distance of about $400 \mathrm{pc}$ and the cluster covers a relatively large sky area. The separation of cluster members from the large population of foreground stars is still incomplete. Clusters at larger distances (1000-2000pc) are concentrated to a smaller field and essentially all stars in these directions are clusters members and thus contamination by foreground stars is low.

Here we present an in-depth study of the young open stellar cluster NGC 2244. Pérez et al. (1987) obtain a cluster distance of $1.67 \mathrm{kpc}$, while a more recent study of the cluster member star V 578 Mon (HD 259135) by Hensberge et al. (2000) points to a distance of $1.39 \mathrm{kpc}$. For our considerations we adopt a mean cluster distance of $1.5 \mathrm{kpc}$. NGC 2244 is embedded in the Rosette Nebula (NGC 2237), which itself is one of the most massive nebulae known. The NGC 2244 and NGC 2237 complex is part of the Mon OB2 association. Here we provide a summary of the major nebulae and cluster properties. For a more detailed compilation we refer the reader to Pérez et al. (1991).

Celnik (1983) estimated that the nebulae consists of $5400 M_{\odot}$ in dust form and $23000 M_{\odot}$ of gas in the ionized cloud (Celnik 1985). The apparent diameter of the 
nebula is about $60^{\prime}$, whereas the star cluster is concentrated to the inner nebula region. NGC 2244 is one of the youngest known open clusters (age 3 Myrs; Massey et al. 1995) and contains 12 bright OB stars of spectral types ranging from $\mathrm{O} 4$ to $\mathrm{B} 2$ within a region of less than $30^{\prime} \times 30^{\prime}$ (Pérez et al. 1987). These OB stars are estimated to contribute about $99 \%$ of the bolometric luminosity of the entire stellar cluster (Cox et al. 1990). While Pérez et al. (1991) claim that stars with $M<4.0 M_{\odot}$ do not exist in NGC 2244 - directly contradicting that star formation and the initial mass function is universal - the H-R diagram in Massey et al. (1995) provides dozens of stars of intermediate spectral types in a pre-main sequence (PMS) phase. Only little is known about late-type stars in this young region, nevertheless, they must exist.

The interstellar reddening to NGC 2244 is low, however, reddening due to the nebula gas has to be taken into account. Massey et al. (1995) report an average cluster reddening of $E(B-V)=0.48$, individual reddening values for stars in NGC 2244 are determined in the range $E(B-V)=0.08 \ldots 0.98$. The differential reddening across the cluster is obviously a result of the surrounding nebula gas. We will further address this point in Sects. 2.1 and 3 where we present and discuss our X-ray results.

ROSAT X-ray observations of NGC 2244 provide over 130 unclassified X-ray sources in the direction of this cluster. Since the spatial distribution of these X-ray sources peaks at the center of the cluster these X-ray emitters should be associated with optically faint cluster members. Note that the number of nearby foreground stars is negligible as we will show in the present work. Optical photometry of these objects will allow us to place them on the H-R diagram and determine if they have yet to arrive on the main sequence. These X-ray detections and the low age of the cluster made it an ideal candidate to study cluster evolution and evolution onto the main sequence.

In order to study the population of active late-type and PMS stars in NGC 2244 we processed archival ROSAT PSPC and HRI observations (presented in Sect. 2.1) and we conducted optical photometry with the Kitt Peak MOSAIC Imager. The optical observations and data reductions are presented in Sect. 2.2. In Sect. 3 we discuss the results and focus on the $138 \mathrm{X}$-ray-selected sources. We present the identifications and a summary table of X-ray and optical properties (Sect. 3.1), color-color diagrams in Sect. 3.2 and use the derived ratio of $\mathrm{X}$-ray to optical luminosity to help distinguish the nature of the $\mathrm{X}$-ray emitters in Sect. 3.4. Lastly, we discuss the evolutionary states of the active stars and implications for star formation processes in Sect. 4.

\section{Observations}

\subsection{ROSAT observations}

We processed archival ROSAT PSPC and HRI data of the Rosette Nebula to search for discrete X-ray sources in the central cluster and the surrounding nebula. Standard
Table 1. ROSAT observation log.

\begin{tabular}{llcc}
\hline \hline ROR & detector & date & exposure (s) \\
\hline WG200196P & PSPC & Apr. 2, 1992 & 4300 \\
& & Oct. 5, 1993 & 12850 \\
WG202155H & HRI & Apr. 5-11, 1996 & 18800 \\
\hline
\end{tabular}

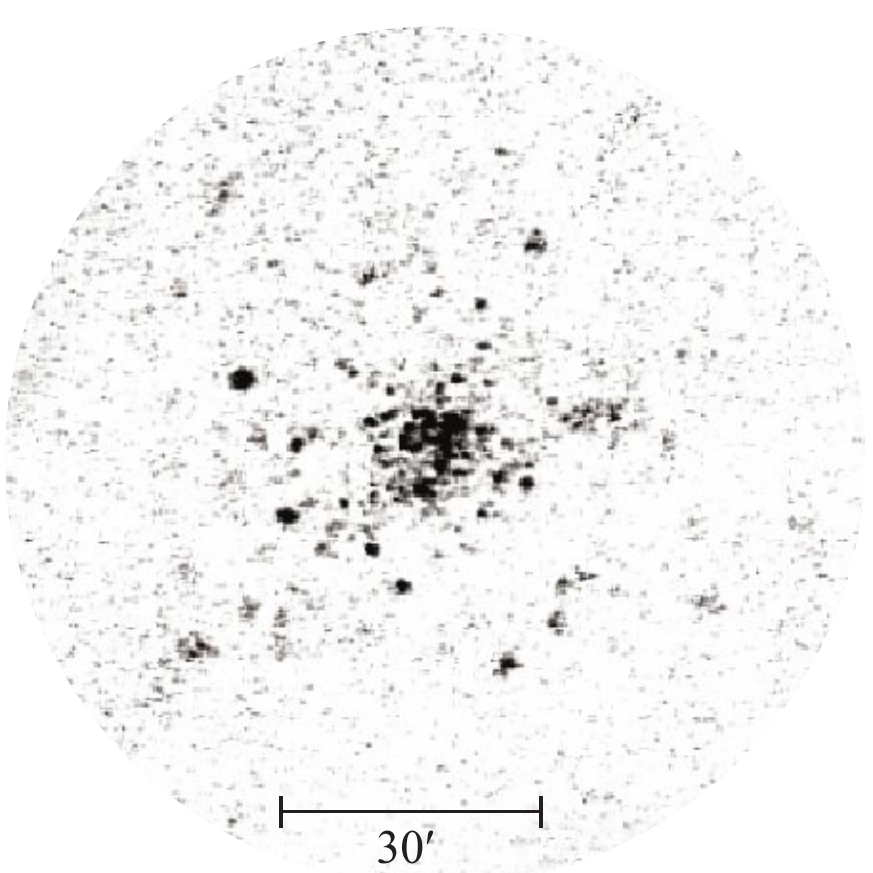

Fig. 1. ROSAT PSPC image in the hard $(0.5-2.0 \mathrm{KeV}$ energy band). The image is centered on NGC 2244.

procedures in the EXSAS software package have been used for data reduction. Table 1 provides a summary of the ROSAT observations included in our analysis, and includes: the ROSAT observation request (ROR) No., the detector (PSPC or HRI), and the date and exposure time of the data set.

In Fig. 1, we show a PSPC image of the Rosette Nebula produced in the hard $(0.5-2.0 \mathrm{KeV})$ energy band. The hard band image demonstrates a large number of X-ray sources in this sky direction. It is worthwhile to mention that the ROSAT observations of the Rosette Nebula do not provide any evidence for diffuse X-ray emission, possibly associated with the nebula or due to an interaction with the stellar cluster.

In the $17 \mathrm{ksec}$ PSPC observation we detected 119 X-ray sources within a radius of $36^{\prime}$ centered on NGC 2244 . The later $18 \mathrm{ksec} H R I$ pointing revealed $47 \mathrm{X}$-ray sources, 28 of them are also detected in both X-ray observations, and 21 PSPC detected X-ray sources lie outside the smaller HRI field of view. As a detection limit a threshold of $4 \sigma$ above the local background count rate has been chosen. In order to test the pointing accuracy of the ROSAT observations we used the optical positions of the early-type stars as listed in the Simbad stellar database to identify the X-ray counterparts in our ROSAT source lists; note that at least the $\mathrm{O}$ stars (e.g. the central star HD 46150) 
and the early B stars are expected to show X-ray emission. In fact, 9 of the X-ray sources detected in the PSPC image are positionally coincident with early type stars of spectral types $\mathrm{O} 4-\mathrm{B} 3,7$ of them are also detected in the HRI image of the cluster. The offsets between optical and X-ray positions of these stars have been chosen to be smaller than $10^{\prime \prime}$. We mention that the nominal on-axis resolution of the PSPC and HRI instruments are $\approx 20^{\prime \prime}$ and $\approx 5^{\prime \prime}$, respectively. Cross correlations of the X-ray and optical positions of these identifications then have been used to minimize boresight errors in the X-ray positions. We obtained $\Delta \mathrm{RA}=2.8^{\prime \prime}, \Delta \mathrm{Dec}=-4.3^{\prime \prime}$ and $\Delta \mathrm{RA}=$ $1.7^{\prime \prime}, \Delta \mathrm{Dec}=2.1^{\prime \prime}$, respectively for the PSPC and HRI pointing. All detected X-ray sources have been corrected appropriately.

As far as the X-ray emission of stars in NGC 2244 is concerned it is important to convert the reddening values into equivalent absorption columns towards the stars. Adopting the average conversion relation $N_{\mathrm{H}}=$ $5.9 \times 10^{21} \mathrm{~cm}^{-2} E(B-V)$, we derive an average column density towards NGC 2244 of $N_{\mathrm{H}}=2.8 \times 10^{21} \mathrm{~cm}^{-2}$ with variations in the range $0.5 \ldots 5.8 \times 10^{21} \mathrm{~cm}^{-2}$. Note that even the lowest reddening observed in NGC 2244 attenuates the X-ray flux in the ROSAT band below $E<0.4 \mathrm{keV}$ by a factor of $\sim 20$. In the case of the PSPC data, a detection in the soft energy band $(E<0.4 \mathrm{keV})$ is thus a clear indicator for an X-ray emitting foreground star. Four $\mathrm{X}$-ray sources are counterparts of stars in the foreground that are listed in Simbad. Based on the detection in the PSPC soft band image below $E<0.4 \mathrm{keV}$ we detected a fifth foreground star among the 138 X-ray emitters. The vast majority are, however, associated with optically fainter stars, and thus requiring sensitive follow-up optical observations we undertook.

\subsection{Optical observations with the KPNO MOSAIC imager}

We observed the young open cluster NGC 2244 with the $8 k \times 8 k$ MOSAIC Imager at Kitt Peak in February 1998 . The MOSAIC Imager when used with the 0.9 meter telescope provides a $1 \mathrm{deg} \times 1 \mathrm{deg}$ field of view. To fully cover the entire Rosette Nebula and regions off the nebula to determine the population of foreground stars, we obtained images at the central cluster position (RA: $06^{\mathrm{h}} 31^{\mathrm{m}} 55.50$ \& Dec: $\left.+04^{\circ} 56^{\prime} 34^{\prime \prime} .31\right)$ and 4 offset positions. For each field 10 dithered exposures were taken in $B V R I$ and $\mathrm{H} \alpha$ filters. While the reductions for the 4 offset fields are still in progress, we present the results of our observations of the central field of NGC 2244 and concentrate on the X-ray selected stars.

The CCD images were processed with the standard procedures in the MSCRED package in IRAF. The astrometry of the MOSAIC images is based on a cross correlation with stars listed in the Tycho catalog. The reduced single frames were then stacked to derive one deep image of NGC 2244 in each of the distinct filters.
Table 2. Summary of optical results.

\begin{tabular}{lcc}
\hline \hline Filter & Number & Limit \\
\hline$B$ & 4879 & 19.5 \\
$V$ & 8550 & 19.5 \\
$R$ & 15618 & 19.8 \\
$I$ & 25615 & 19.5 \\
\hline
\end{tabular}

In order to search for objects in our images and to derive magnitudes we ran SExtractor (version 2.1) on the images. A $5 \sigma$ threshold above the combined sky and nebula background was chosen for secure object detection. Stars in the field with existing photometric data were used to calibrate our observations. Extended objects, presumably extragalactic background objects, were excluded from the analysis. We show the dithered and combined $R$ band image of the central portion of NGC 2244 in Fig. 2.

Results from the central field yielded over 25000 stars in the $I$ band. Table 2 summarizes the number of detections in selected filters and limiting magnitudes.

To demonstrate the quality of our optical data, Fig. 3 provides the errors of the magnitudes derived in the $V$ band. As can be seen these errors are $<0.02 \mathrm{mag}$ in general for stars brighter than $V<17 \mathrm{mag}$ and do not become appreciable until $>19$ mag. Note these errors reflect only the internal error and systematic errors might be larger, particularly, at $V<10$ mag when stellar images are saturated. The magnitude errors in the other filter bands are similar to the $V$ band errors.

The absolute calibration of the magnitudes derived in the $B V R I$ filter bands is based on a regression analysis with existing photometry for stars in NGC 2244 listed in Simbad, WEBDA, the Tycho catalog, and in Massey et al. (1995). In Fig. 4 we show the differences between our values and $V$ magnitudes taken from the literature as a function of $V$. For the calibration of the $\mathrm{H} \alpha$ magnitudes we used a flux calibrated high resolution spectrum of the A2V star HD 46107 obtained with AMOS at the Lick $3 \mathrm{~m}$ telescope (Berghöfer \& Christian 2002). The spectrum was folded with the filter transmission to derive the absolute $\mathrm{H} \alpha$ magnitude for this star and correct all measured $\mathrm{H} \alpha$ magnitudes.

\section{Results}

\subsection{Source identifications}

The two source lists with X-ray emitters detected in the ROSAT PSPC and HRI observations have been cross matched to identify identical X-ray sources. For cross identification we allowed a maximum offset of $25^{\prime \prime}$ between PSPC and HRI position. In a few cases the HRI was able to resolve two close $\mathrm{X}$-ray sources that appeared as a single source in the PSPC image. Based on this cross identification we produced a combined X-ray source list, which we used as an input list for a cross identification with our data base of optical photometry for stars in NGC 2244. As maximum offsets between X-ray and optical position 


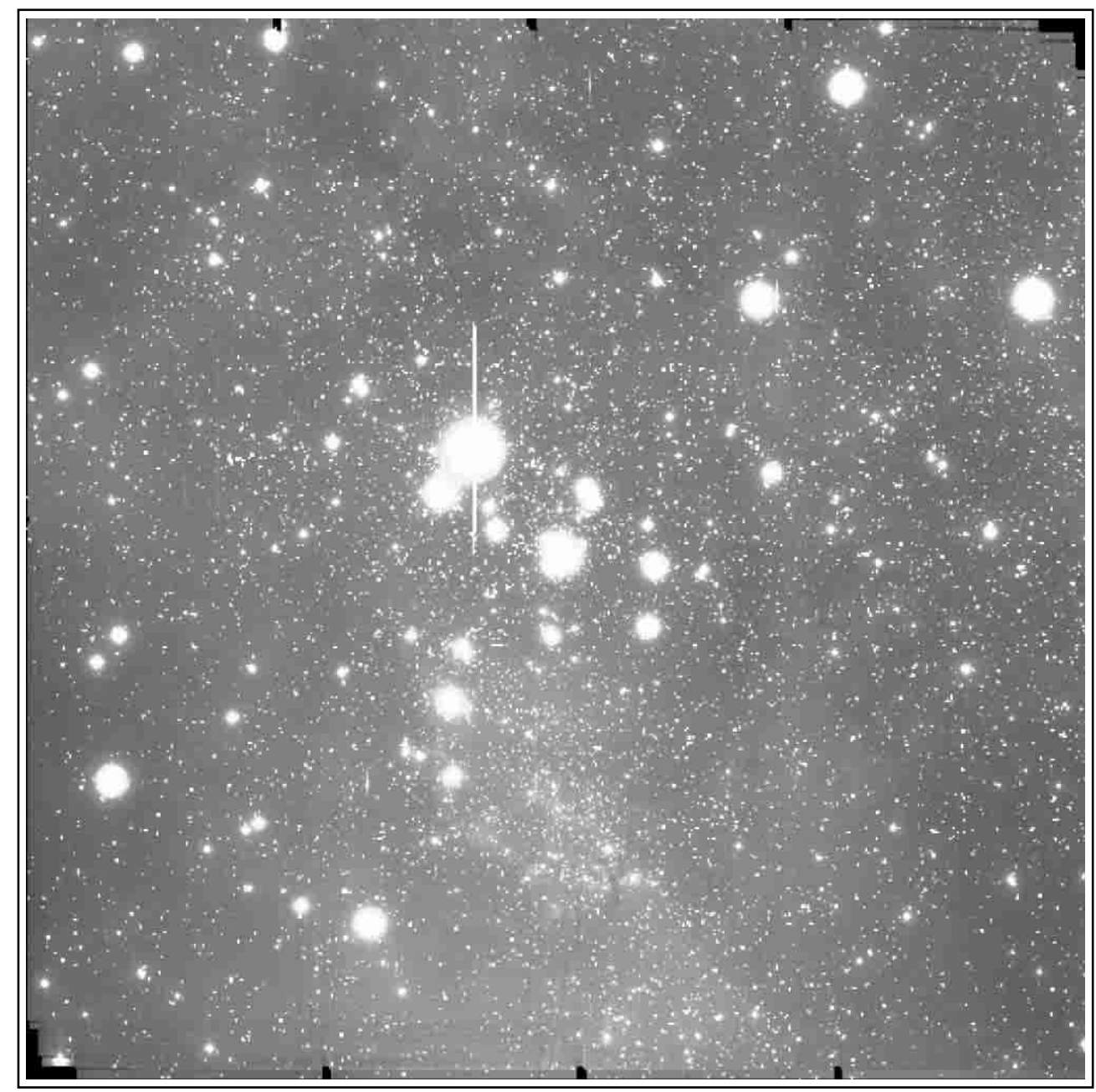

Fig. 2. MOSAIC $R$-band image of NGC 2244. Field size is $1^{\circ} \times 1^{\circ}$.

we allowed $25^{\prime \prime}$ and $5^{\prime \prime}$, respectively for a PSPC and an HRI source. As a preference the brightest optical counter part was accepted to be the right match within the error circle around the X-ray source. In some cases another optically fainter object appeared closer to the X-ray position, which was accepted as an alternative identification. Note that the preference to the optically brightest object may result in some missidentifications in the range of late $\mathrm{B}$ and early A stars. However, these stars can also be unresolved binaries with late-type companions. For a detailed discussion of X-ray emission from such stars, which actually should be avoid of X-ray emission, we refer to Berghöfer \& Schmitt (1994b). All identifications have been carefully checked by a visual inspection of the X-ray and optical images.

We present the optical and X-ray results for the $138 \mathrm{X}$ ray sources with high quality photometric information in Table 3. Table 3 includes the sources J2000 optical positions and offset to the most accurate X-ray positions (HRI position in case of an HRI detection, otherwise PSPC position), HRI and PSPC count rates and derived luminosities and the MOSAIC $B V I R \& \mathrm{H} \alpha$ magnitudes. Remarks and comments about source identifications, source confusion or other information are given in the last column of the Table. In two cases with no optical counter part within the error circle around the X-ray position, the X-ray position is listed in Table 3 .

\subsection{X-ray properties}

In order to test for long term variability and for a consistency check we first compare the derived PSPC and HRI $\mathrm{X}$-ray luminosities and upper limits of the X-ray sources covered by both observations. The PSPC and HRI derived X-ray luminosities of the detected early-type stars are consistent within the errors. This result confirms earlier long-term variability studies of early-type stars, which showed that early-type stars are rather constant X-ray emitters (e.g., Berghöfer \& Schmitt 1994a). Furthermore, this demonstrates that the PSPC and HRI observations of NGC 2244 are consistent.

Only in the case of the eclipsing binary HD 259135, consisting of two early B components within a 2.4 days orbit (Hensberge et al. 2000), we find evidence for variability in an early-type star. During the PSPC observation this star showed an $\approx 10$ times larger flux than during the later 


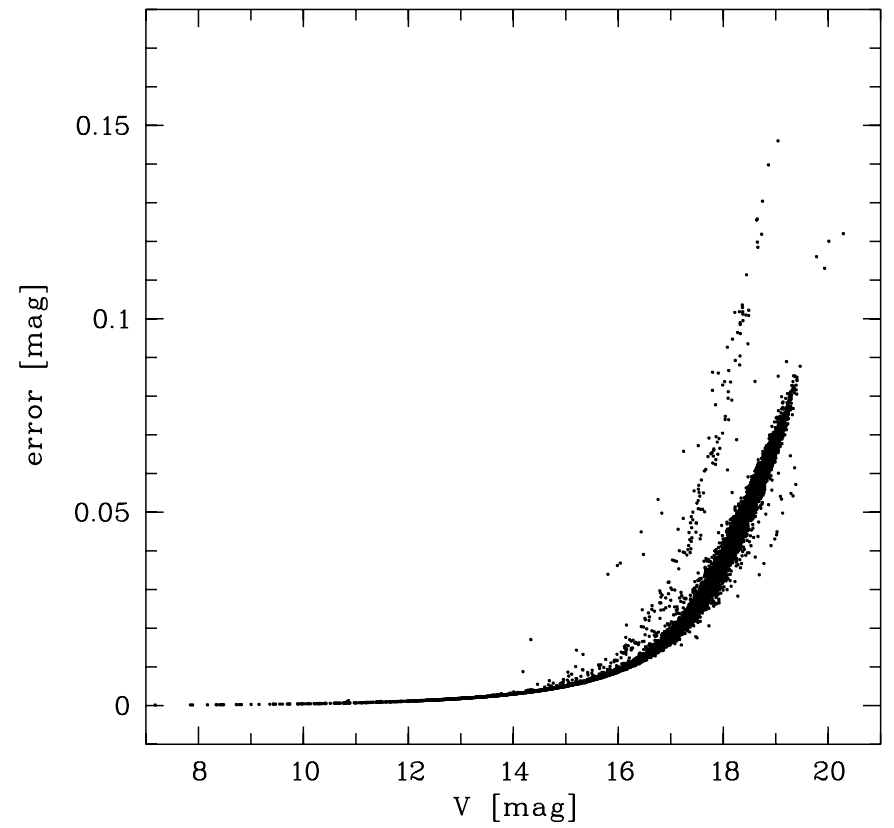

Fig. 3. As an example the photometric error as a function of magnitude is shown for all stars detected in the combined $V$ band image. The second (smaller) distribution of stars to the left of the main distribution is due to different exposure times in the final stacked image at the position of gaps in the single frames.

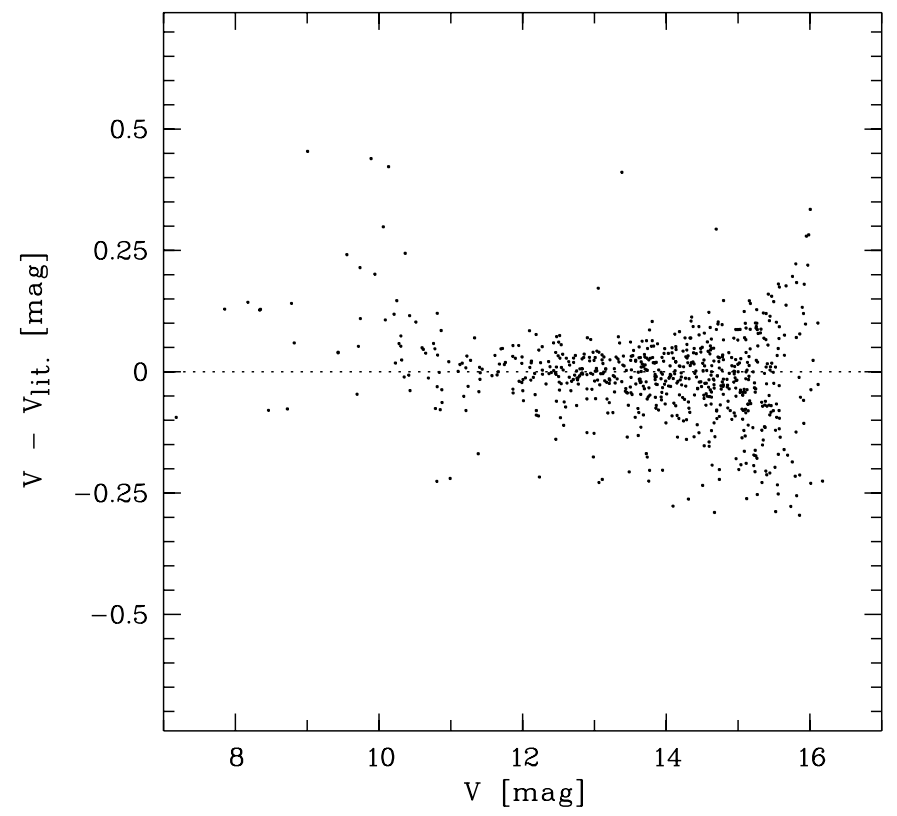

Fig. 4. Deviations of our $V$ band photometry from values obtained in previous studies of NGC 2244 plotted as a function of $V$ magnitude. The large deviations in the range $V<10$ result from saturation of stellar images.

HRI observation. It is suggestive that the observed X-ray variability is related to the binary nature of this star. For all other X-ray emitters we find that long term variations in X-ray flux are smaller than a factor of $\approx 10$, similar to variations of the solar X-ray flux.

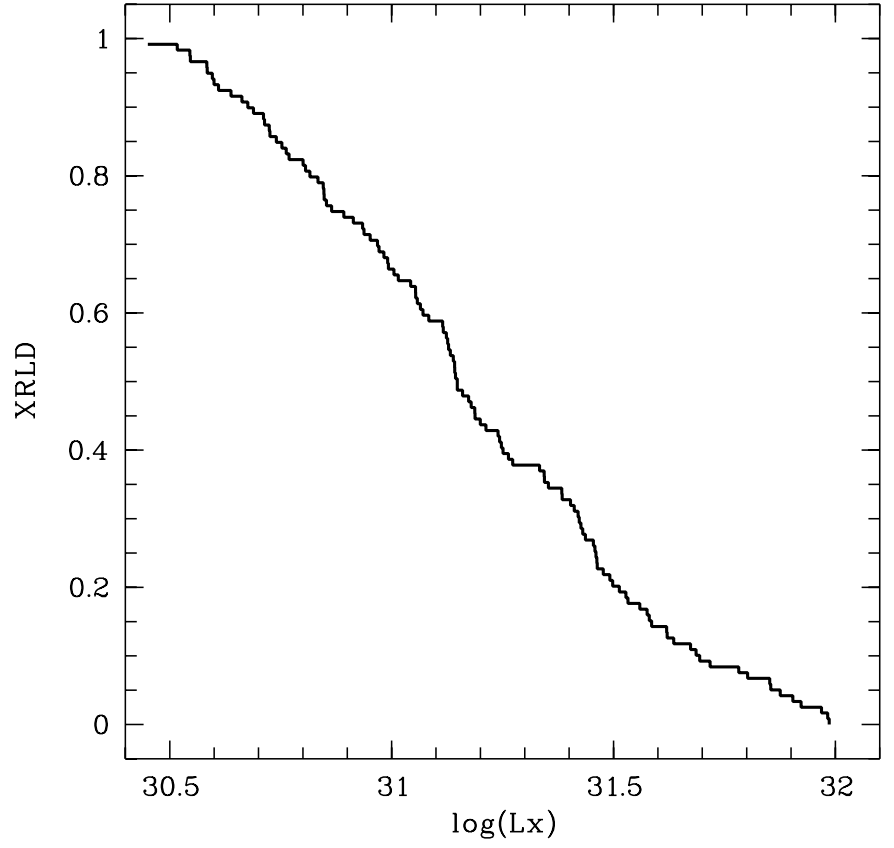

Fig. 5. X-ray luminosity distribution function of the X-ray emitters in NGC 2244, excluding foreground stars and earlytype stars in the cluster.

In order to convert count rates into X-ray luminosities we adopted a model spectrum of a thermal plasma (Raymond-Smith 1977) with a temperature of $1.0 \mathrm{keV}$, typical of late-type star X-ray emission, simulated energy conversion factors for the effective areas of the ROSAT PSPC and HRI, and a mean cluster distance of $1.5 \mathrm{kpc}$. These simulations take into account interstellar foreground absorption of $N_{\mathrm{H}}=3 \times 10^{21} \mathrm{~cm}^{-2}$. We derive conversion factors of $2.5 \times 10^{-11} \mathrm{erg} \mathrm{cm}^{-2}$ count $^{-1}$ and $6.0 \times 10^{-11} \mathrm{erg} \mathrm{cm}^{-2}$ count $^{-1}$, respectively for the PSPC and the HRI.

Next we investigate the X-ray luminosities of the X-ray emitters detected in the PSPC observation of NGC 2244, excluding stars that are identified as early-type stars or foreground stars. In Fig. 5 we plot the respective cumulative X-ray luminosity distribution function. The observed X-ray luminosities range from $\log \left(L_{\mathrm{x}}\right)=30.5$ up to 32.0 , which is a clear indicator for very active late-type PMS stars (e.g., Wichmann et al. 1996; Gagné et al. 1995). Note that late-type dwarfs usually exhibit significantly lower X-ray luminosities below $\log \left(L_{\mathrm{x}}\right) \approx 29$ (Schmitt 1997).

\subsection{Color-magnitude diagrams}

We constructed a color-magnitude diagram using $V$ and $B-V$ colors (Fig. 6). Stars detected in the ROSAT images are marked with diamonds. The solid line represents the zero-age main-sequence (ZAMS) for the NGC 2244 cluster; a reddening to the cluster of $E_{B-V}=0.48$ and a distance modulus of 12.26 (WEBDA open cluster database, Lausanne; Massey et al. 1995) is adopted. Note that Massey et al. (1995) report large variations in the 
reddening towards the early-type stars in NGC 2244. They provide minimum and maximum values for the reddening of $E_{B-V}=0.38$ and 0.85 , whereas the individual measurements vary in the range $0.08 \leq E_{B-V} \leq 0.98$. The two dashed lines in Fig. 6 provide the location of the ZAMS for reddenings of $E_{B-V}=0.38$ and 0.85 , the dotted lines correspond to $E_{B-V}=0.08$ and 0.98 .

Taking into account the differential cluster reddening, the early-type stars in our sample are located close to or to the right of the ZAMS. This is expected due to the short PMS phase of early-type stars and confirms that our results are consistent with the spectroscopic results by Massey et al. (1995).

For stars fainter than $V \simeq 14$ (spectral type later than $\approx$ mid $A$ ) the color-magnitude diagram shows a larger spread in $B-V$ color. Stars to the left of the ZAMS plotted for $E_{B-V}=0.08$ are assumed to be stars in the foreground of NGC 2244. The bulk portion of the fainter stars is concentrated in the color range $0.7 \approx B-V \approx 1.3$. An individual dereddening of these stars requires optical spectroscopy and cannot be done within the analysis of our photometry data. However, the deviations of the points from the ZAMS are in most cases explainable by reddening values that are compatible with those found in previous studies for this field. A significant number of stars are located in the color-magnitude diagram to the right of the ZAMS when assuming a reddening of $E_{B-V}=0.98$. This may show that these stars are significantly younger than the average cluster member in NGC 2244 or even more embedded into the nebula and exhibiting even higher reddening values, which we cannot distinguish on the basis of existing data. Apart from this discussion it is clear that the optically fainter low-mass stars in the 3 Myrs old cluster NGC 2244 must be in a PMS phase.

To further investigate the evolutionary status of the late-type stars in NGC 2244, we take a closer look at the low-mass stars in the color-magnitude diagram in Fig. 7. For comparison the ZAMS and isochrones representing stellar ages of $0.1,0.5,1$, and $3 \mathrm{Myrs}$ are plotted assuming the average cluster reddening of $E_{B-V}=0.48$. The isochrones are based on the model computations of D'Antona \& Mazzitelli (1994). Adopting the tabulated stellar parameters and photometry values in SchmidtKaler (1982) the isochrones have been converted from the theoretical plane into the plane of the color-magnitude diagram. As can be seen, most of the stars are located to the left of the 3 Myrs isochrone, which is offset to the ZAMS by about $0.4 \mathrm{mag}$ in $B-V$. When assuming a reddening in the range $0.08 \leq E_{B-V} \leq 0.98$ approximately half of the stars are consistent with an age of $\approx 3$ Myrs. Within the range of observed cluster reddening vastly all X-ray emitters detected in NGC 2244 are consistent with an age of $\approx 3$ Myrs.

However, even when assuming lowest cluster reddening about $45 \%$ of the cluster stars are located in between the ZAMS and the 3 Myrs isochrone thus indicating an age significantly older than 3 Myrs. This finding is suggestive of an earlier star formation phase in or near the Rosette

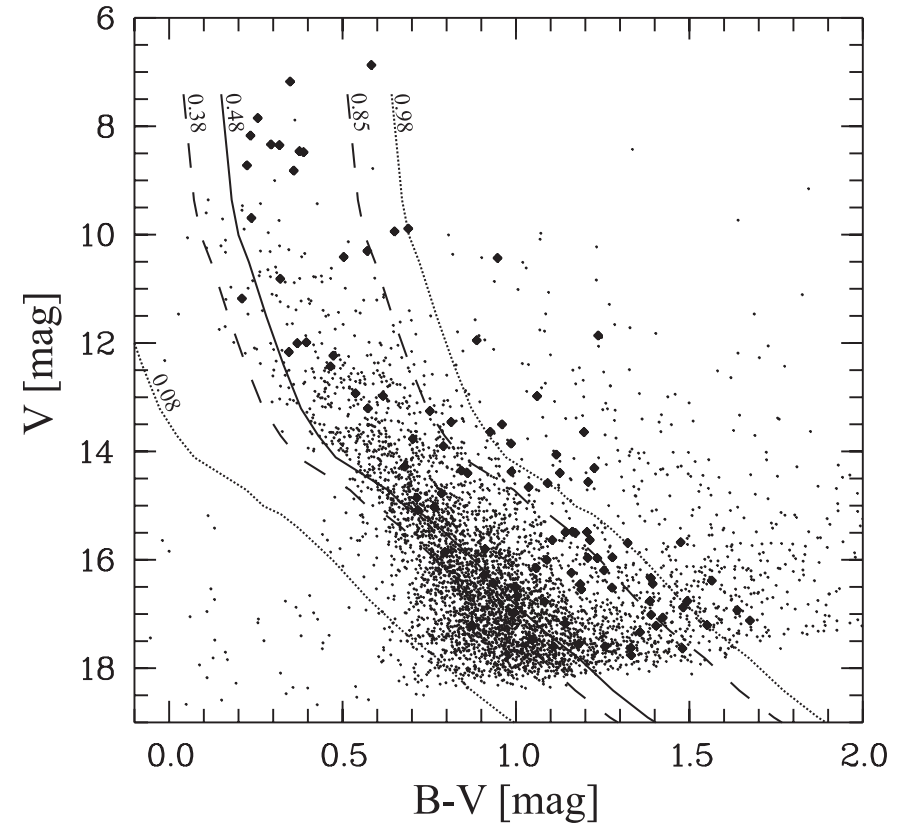

Fig. 6. Color-magnitude diagram of the stars in NGC 2244. The X-ray detected stars are marked with larger diamonds. The solid line provides the zero-age main-sequence (ZAMS) assuming a distance modulus of 12.26 and the average cluster reddening of $E_{B-V}$ of 0.48 . The dashed lines represent the cluster ZAMS for the lower and upper reddening limits of $E_{B-V}=0.38$ and 0.85 , whereas the dotted lines reflect the ZAMS location assuming the minimum $\left(E_{B-V}=0.08\right)$ and maximum $\left(E_{B-V}=0.98\right)$ reddening measured for individual stars in NGC 2244 (Massey et al. 1995).

Nebula/NGC 2244 complex. We will further discuss these older stars in Sect. 4. Additionally, stars exist to the right of the 3 Myrs isochrone when adopting $E_{B-V}=0.98$. This demonstrates that stars significantly younger than 3 Myrs exist, star formation is still in progress, and continuous star formation likely has happened (cf. Pérez et al. 1987, 1989)

PMS stars are known to be very active stars showing strong X-ray and $\mathrm{H} \alpha$ emission. For our sample of stars we now explore the $\mathrm{H} \alpha$ properties. To be able to search for $\mathrm{H} \alpha$ emission, the magnitudes in $\mathrm{H} \alpha$ need to be normalized to the continuum flux. According to Sung et al. (2000) the combined quantity $\mathrm{H} \alpha-(V+I) / 2$ provides a good measure for the flux in the $\mathrm{H} \alpha$ line. We employed a high resolution optical spectrum of the A2V star HD 46107 (No. 23 in Table 3) taken with AMOS at the Lick $3 \mathrm{~m}$ telescope and the observed photometry of this star to calibrate $\mathrm{H} \alpha-$ $(V+I) / 2$. We find that $\mathrm{H} \alpha-(V+I) / 2=+0.07$ provides the dividing line between $\mathrm{H} \alpha$ emission and absorption. This value includes a small correction of the weak $\mathrm{H} \alpha$ absorption in the spectrum of this A star.

To study a large dynamic range, we plot the $\mathrm{H} \alpha-(V+$ $I) / 2$ vs. $V-I$ colors in Fig. 8 . Here we have also included the 4093 stars with detections in our $V, R, I$, and $\mathrm{H} \alpha$ images and indicated the X-ray emitters as diamonds. The majority of X-ray emitters as well as a significant fraction of the non X-ray emitters have very strong $\mathrm{H} \alpha$ emission 


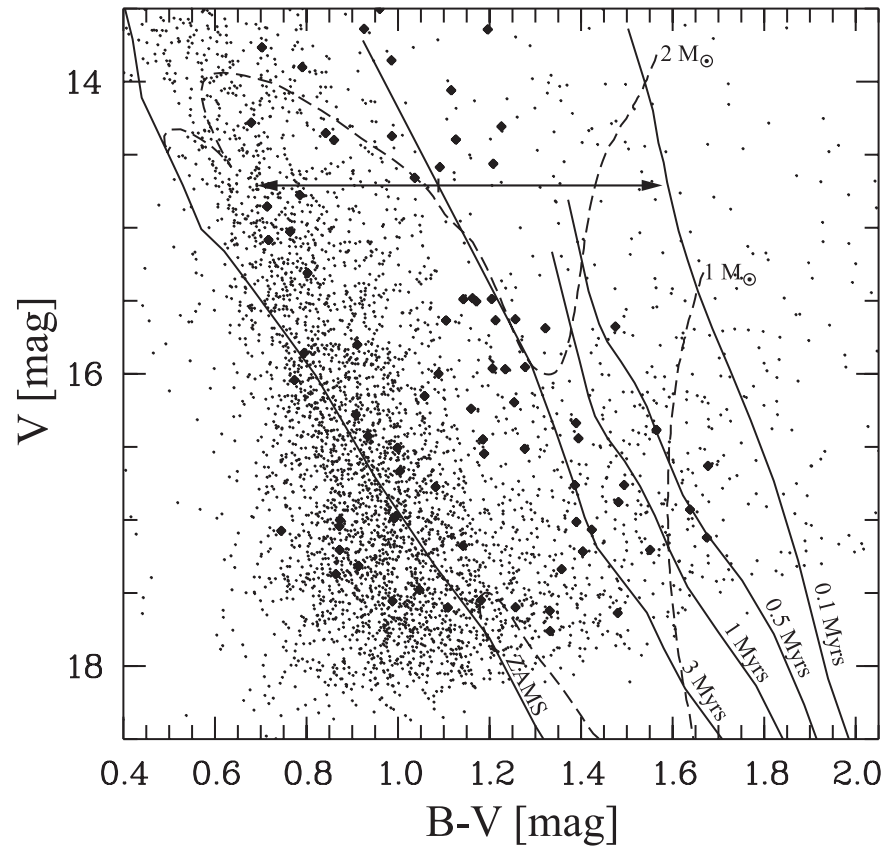

Fig. 7. Color-magnitude diagram of the lower mass stars in NGC 2244. The X-ray detected stars are marked with diamonds, the solid lines represent the ZAMS and isochrones for stellar ages of $0.1,0.5,1$, and 3 Myrs (see text). The dashed lines provide evolutionary tracks for stars with stellar masses of 1.0 and $2.0 M_{\odot}$. An average cluster reddening of $E_{B-V}=0.48$ has been assumed. The horizontal bar with arrows indicates the range of reddening observed for stars in this cluster.

and show up at values less than 0.07 on the ordinate. This again confirms that these stars are in the PMS phase.

\subsection{X-ray to optical luminosities}

We now investigate the properties of our 138 X-ray emitters by comparing their X-ray to optical luminosities. Berghöfer et al. (1997) and previous studies have well established that X-ray emission of early-type stars obeys the relation $L_{\mathrm{x}} / L_{\mathrm{bol}} \sim 10^{-7}$. and late-type stars have ratios on the order of $L_{\mathrm{x}} / L_{\mathrm{bol}} \sim 10^{-6}$ to $10^{-3}$ (Mathioudakis et al. 1995; Fleming et al. 1995).

In Fig. 9 we show the ratio of the X-ray to optical luminosity plotted against the $V$ magnitude. This figure shows a remarkable correlation of increased X-ray luminosity for the fainter cluster members and indicates these are young, coronally active stars. For stars fainter than $V \stackrel{\sim}{\sim} 14$ we find $f_{\mathrm{x}} / f_{\mathrm{v}}$ ratios in the range $10^{-2.5}$ to $10^{0.5}$, which translates in $L_{\mathrm{x}} / L_{\text {bol }} \approx 10^{-2}$ to $10^{-3}$ when assuming bolometric corrections listed in SchmidtKaler (1982). The optically bright OB stars with $f_{\mathrm{x}} / f_{\mathrm{v}} \approx$ $10^{-3.7}$ and $V \approx 10$ are consistent with earlier analyses and result in $L_{\mathrm{x}} / L_{\mathrm{bol}} \sim 10^{-7}$ (Chlebowski et al. 1989; Berghöfer et al. 1997). A group of roughly 20 stars falls in the range $V=10-14$. These stars should be of spectral types mid B to A. Since no X-ray production mechanism is know to generate $\mathrm{X}$-ray emission in such stars they should be devoid of any X-ray emission (cf.

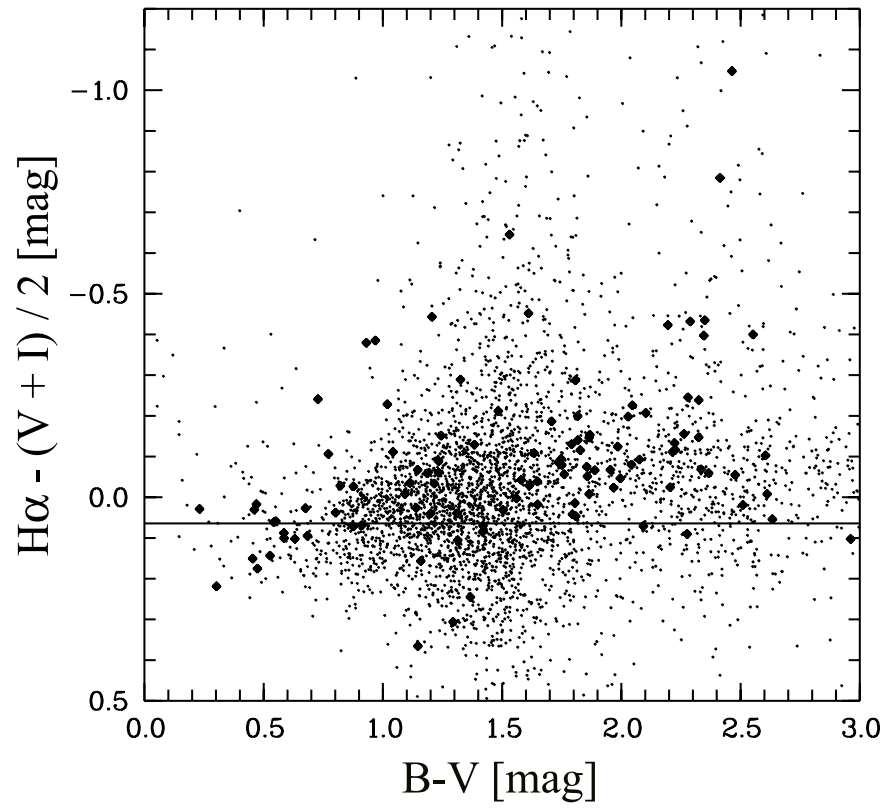

Fig. 8. $\mathrm{H} \alpha-(V+I) / 2$ versus $V-I$ diagram of NGC 2244 . All secure photometric detections are included. X-ray selected stars are marked with larger diamonds (see text).

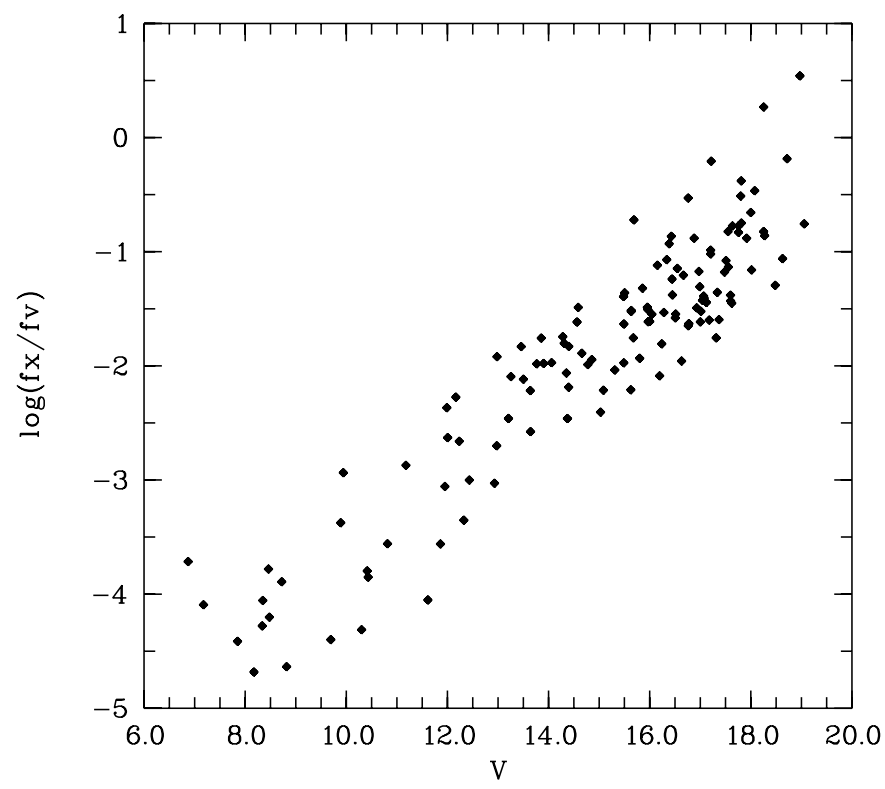

Fig. 9. $\log \left(f_{\mathrm{x}} / f_{\mathrm{v}}\right)$ vs. $V$ diagram of NGC 2244 .

Berghöfer \& Schmitt 1994b and references therein). According to the standard hypothesis these stars do have unresolved late-type companions that are responsible for the X-ray emission.

The average luminosities of our sample are on the higher end for active late-type stars, with $L_{\mathrm{x}} \sim 7 \times$ $10^{30} \mathrm{erg} \mathrm{s}^{-1}$ and $L_{\mathrm{x}} / L_{\text {bol }} \approx 10^{-2}$ to $10^{-3}$; late-type dwarfs usually produce soft X-ray emission with luminosities below $10^{30} \mathrm{erg} \mathrm{s}^{-1}$. However, this is in agreement with findings for young PMS late-type stars in star forming regions (e.g., in Orion); Gagné et al. (1995) report X-ray luminosities up to several $10^{31} \mathrm{erg} \mathrm{s}^{-1}$ for PMS stars in Orion. 


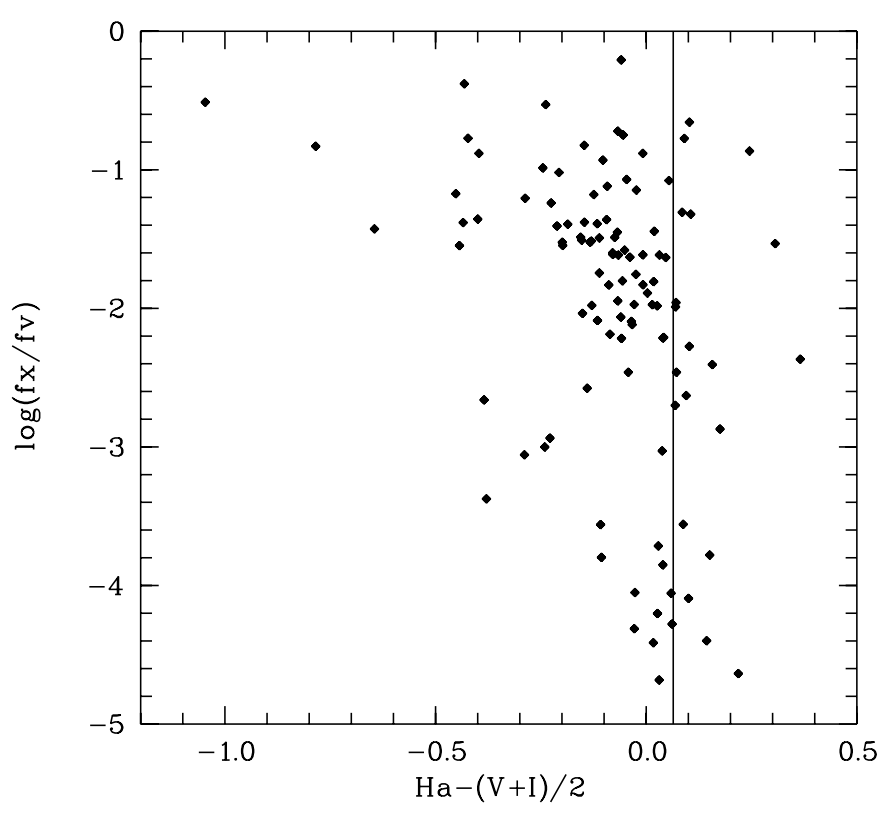

Fig. 10. $\log \left(f_{\mathrm{x}} / f_{\mathrm{v}}\right)$ vs. H $\alpha$ diagram of NGC 2244. The vertical line indicates the dividing line between $\mathrm{H} \alpha$ emission and absorption.

In Fig. 10 we provide a plot of the X-ray-to-optical fluxes $f_{\mathrm{x}} / f_{\mathrm{v}}$ as a function of $\mathrm{H} \alpha$. This plot shows a very strong clustering among the late-type stars at $f_{\mathrm{x}} / f_{\mathrm{v}} \sim$ $10^{-1.5}$ to $10^{-2}$ and weak $\mathrm{H} \alpha$ emission. For larger $f_{\mathrm{x}} / f_{\mathrm{v}}$ ratios the spread in $\mathrm{H} \alpha-(V+I) / 2$ magnitudes is most extreme, reaching from the absence of $\mathrm{H} \alpha$ emission up to strong $\mathrm{H} \alpha$ emission. This result is consistent with findings obtained for late-type stars in the solar neighborhood (Mathioudakis \& Doyle 1991). However, since the $\mathrm{X}$-ray and optical observations were taken at two different epochs we cannot exclude that significant time variability in the X-ray and $\mathrm{H} \alpha$ properties among the latest spectral types appear and more massive late-type stars (spectral types late $\mathrm{A}-\mathrm{F}$ ) show rather constant $\mathrm{X}$-ray-tooptical properties. A second but smaller group of stars can be found at $f_{\mathrm{x}} / f_{\mathrm{v}} \approx 10^{-4}$. These stars can be identified as the early-type stars in our sample, which are scattered around the dividing line between $\mathrm{H} \alpha$ emission and absorption. A lower number of stars with $\mathrm{H} \alpha$ in emission appears near $f_{\mathrm{x}} / f_{\mathrm{v}} \sim 10^{-3}$. Two of these stars are identified to be (late-type) foreground stars, the other four objects have $V$ magnitudes in the range $V=12.8 \ldots 14.4 \mathrm{mag}$. These objects are presumably PMS stars of intermediate spectral types, e.g. Herbig Ae/Be stars.

\section{Conclusions}

We have used the Kitt Peak MOSAIC wide field Imager to obtain high-resolution optical photometry of the very young cluster NGC 2244 in the Rosette Nebula. Based on these data we optically identified 138 X-ray sources, which we detected in archival ROSAT PSPC and HRI images of this cluster. Optical and X-ray properties of the $\mathrm{X}$-ray and non-X-ray emitters were compared. The optical photometry allowed us to study the location of the cluster stars in the color-magnitude diagram well into the range of K spectral types.

An accurate age dating for the individual stars is difficult to achieve due to the large differential reddening across the Rosette Nebula region and additional spectroscopic observations are required. However, our wide field imaging photometry of NGC 2244 demonstrates that many of the cluster stars must be in a PMS phase. Furthermore, we find that roughly $50 \%$ of the fainter cluster members are consistent with an age of $\approx 3$ Myrs. Even taking into account lowest cluster reddening a significant fraction of stars is located closer to the ZAMS than to the 3 Myrs isochrone. Therefore, the age of these stars should be significantly older than 3 Myrs. According to earlier studies of the early-type stars in the direction of the Rosette Nebula, up to $30 \%$ of these stars are found to be non-members of NGC 2244 (see Pérez et al. 1991 and references therein). Pérez et al. suggested that these stars are surrounding NGC 2244 and are members of the Mon OB2 association. This might also be the case for the stars of later spectral types that appear to be older than 3 Myrs in this study.

Additionally, about $5 \%$ of the stars are far off to the right of the 3 Myrs isochrone. Assuming maximum cluster reddening cannot explain their location in the colormagnitude diagram and suggests that these stars are significantly younger than 3 Myrs and have been formed quite recently. This indicates that the Rosette Nebula is still an active star forming region. However, we cannot completely exclude that these stars are even deeper embedded in the nebula and the apparently younger age is a result of even higher reddening. On the other hand our results confirm earlier reports of continuous star formation in the Rosette Nebula (Pérez et al. 1987, 1989).

A large fraction of the late-type stars in NGC 2244 shows $\mathrm{H} \alpha$ emission. In the case of the X-ray emitters among these stars, the majority are also $\mathrm{H} \alpha$ emitters. For the late-type cluster members the observed X-ray luminosities and X-ray to optical luminosity ratios of $L_{\mathrm{x}} \sim 7 \times 10^{30} \mathrm{ergs}^{-1}$ and $L_{\mathrm{x}} / L_{\mathrm{bol}} \approx 10^{-2}$ to $10^{-3}$ demonstrate activity levels at the upper end so far observed for late-type PMS stars. The lower number of early-type stars in the Rosette Nebula shows X-ray properties consistent with the canonical value of $L_{\mathrm{x}} / L_{\mathrm{bol}} \sim 10^{-7}$.

The comparison of the $\mathrm{H} \alpha$ properties with the X-ray to optical flux ratios show substantially different $\mathrm{H} \alpha$ properties ranging from the absence of $\mathrm{H} \alpha$ emission to strong emission for the latest spectral types ( $\mathrm{K}-\mathrm{M}$ stars). More massive late-type stars provide more constant fluxes in the $\mathrm{H} \alpha$ line and in the X-rays. Mathioudakis \& Doyle (1991) report a similar behavior among late-type stars in the solar neighborhood. Since no definite explanation exists for this observation we can only speculate that variability in the $\mathrm{H} \alpha$ line and the $\mathrm{X}$-ray flux, which were not simultaneously observed, lead to the large differences in the activity indicators for the latest spectral types observed. 
In summary, with our combined X-ray and wide field optical photometry study of the stellar content in the NGC 2244/Rosette Nebula region we were able to identify activity PMS stars in this young and active region in our Galaxy. Further spectroscopic observations shall be carried out to obtain physical parameters for selected latetype PMS stars in this cluster independent of reddening effects resulting from the surrounding nebula.

Acknowledgements. We thank F. Valdes (NOAO) for his quick help with the MSCRED software package. We acknowledge useful discussions with Michael Sterzik, Michael Freyberg, Jürgen Schmitt, and Marc Hempel. TWB was supported in part by a Feodor-Lynen Fellowship of the Alexander von Humboldt-Stiftung. This research has made use of the Simbad stellar database.

\section{References}

Berghöfer, T. W., Schmitt, J. H. M. M., Danner, R., \& Cassinelli, J. P. 1997, A\&A, 322, 167

Berghöfer, T. W., \& Schmitt, J. H. M. M. 1994a, A\&A, 290, 435

Berghöfer, T. W., \& Schmitt, J. H. M. M. 1994b, A\&A, 292, L5

Berghöfer, T. W., Schmitt, J. H. M. M., \& Cassinelli, J. P. 1996, A\&AS, 118, 481

Celnik, W. E. 1983, The Messenger (ESO), No. 32, 23
Celnik, W. E. 1985, A\&A, 144, 171

Chlebowski, T., Harnden, F. R., \& Sciortino, S. 1989, ApJ, 341,427

Cox, P., Deharveng, L., \& Leene, A. 1990, A\&A, 230, 181

D'Antona, F., \& Mazzitelli, I. 1994, ApJS, 90, 467

Fleming, T. A., Schmitt, J. H. M. M., \& Giampapa, M. 1995, ApJ, 450, 401

Gagné, M., Caillault, J.-P., \& Stauffer, J. R. 1995, ApJ, 445, 280

Hensberge, H., Pavlovski, K., \& Verschueren, W. 2000, A\&A, 358,553

Massey, P., Johnson, K. E., \& De Gioia-Eastwood, K. 1995, ApJ, 454, 151

Mathioudakis, M., \& Doyle, J. G. 1991, A\&A, 244, 409

Mathioudakis, M., Fruscione, A., Drake, J. J., et al. 1995, A\&A, 300, 775

Pérez, M. R., The, P. S., \& Westerlund, B. E. 1987, PASP, 99, 1050

Pérez, M. R., Joner, M. D., The, P. S., \& Westerlund, B. E. 1989, PASP, 101, 195

Pérez, M. R. 1991, Rev. Mex. Astron. Astrofis., 22, 99

Raymond, J. C., \& Smith, B. W. 1977, ApJS, 35, 419

Schmidt-Kaler, T. 1982, in Landolt-Börnstein VI/2b (1982), 451

Schmitt, J. H. M. M. 1997, A\&A, 318, 215

Sung, H., Chun, M.-Y., \& Bessell, M. S. 2000, ApJ, 120, 333

Wichmann, R., Krautter, J., Schmitt, J. H. M. M. et al. 1996, A\&A, 312, 439 